\title{
Editorial
}

\section{Modeling and Processing of Radar Signals for Earth Observation}

\author{
Maria Greco ${ }^{1}$ and Simon Watts ${ }^{2}$ \\ ${ }^{1}$ Dipartimento di Ingegneria dell'Informazione, Università di Pisa, 56122 Pisa, Italy \\ ${ }^{2}$ Thales Aerospace Division, Manor Royal, Crawley, West Sussex RH10 9HA, UK
}

Correspondence should be addressed to Maria Greco, m.greco@iet.unipi.it

Received 14 October 2008; Accepted 14 October 2008

Copyright (c) 2008 M. Greco and S. Watts. This is an open access article distributed under the Creative Commons Attribution License, which permits unrestricted use, distribution, and reproduction in any medium, provided the original work is properly cited.

The rapidly growing number of real aperture and synthetic apertureradars (SARs) devoted to Earth observation provides today a very broad coverage across space, time, and the electromagnetic spectrum. Very large quantities of data and images are being systematically collected, processed, and stored. The all-weather, day, and night capabilities of these radar sensors permit acquisition of information under conditions that are not possible with EO sensors. Radars can monitor iceberg position, movement, and age to improve safety at sea. They can provide useful information on oceans and their currents. Radars can also explore vast areas of the Earth, providing an inventory of potential mineral resources, new transportation routes, freshwater supplies, sites for agriculture, and so on.

The papers in this special issue reflect some of the many varied applications of radar that are being researched today.

Synthetic aperture radar, whether from space or airborne platforms, continues to attract much attention. Spatial resolutions of the order of $1 \mathrm{~m}$ are currently available from spacebased SAR systems such as TerraSAR-X, whilst airborne spotlight SAR systems can achieve resolutions of the order of $10 \mathrm{~cm}$. Many space-based radars are now fully polarimetric, and there is considerable scope for interferometric SAR operation, either using repeat-pass methods or tandem platforms. In their paper entitled "Scattering-based model of the SAR signatures of complex targets for classification applications," G. Margarit and J. J. Mallorqui present a method for analysing SAR imagery and the scattering from complex targets. This has led to a proposed method for classifying targets such as ships and urban buildings, using features obtained from polarimetric and interferometric SAR images.
Interferometric techniques can also be applied to analyse the movement of targets in an SAR image. A. Budillon et al. describe a technique for detecting radially moving targets in an SAR image in their paper "Multi-channel alongtrack interferometric SAR systems: Moving targets detection and velocity estimation." They consider the performance of multichannel AT-InSAR systems in terms of moving target detection and the accuracy of radial velocity estimation.

Polarimetric data can also be successfully used for classification purposes in inverse synthetic aperture radar (ISAR) images, as described by M. Martorella et el. in "CLEAN technique for polarimetric ISAR." This paper addresses the problem of estimating the position and the scattering vector of target scattering centres from polarimetric ISAR images. The CLEAN techniques are used for reducing the data size of the images without losing useful information, with the aim of classifying and recognizing objects on the Earth surface.

A very interesting area of research in the last few years concerns the applications of passive SAR bistatic systems. In the paper entitled "Experiences gained during the development of a passive BSAR with GNSS transmitters of opportunity," M. Cherniakov et al. present an overview of the research conducted at the University of Birmingham in the area of space-surface bistatic synthetic aperture radar (SSB-SAR) since 2003. The main aim of the research is to experimentally demonstrate the feasibility and performance of airborne SS-BSAR, utilizing the Global Navigation Satellite System (GNSS) as the transmitter of opportunity. The paper highlights and briefly discusses the various factors that determine image quality, including various systems 
parameters (related to resolution, power budget, etc.), signal processing algorithms (for imaging, synchronization, etc.), and specific problems to be addressed (such as interference and motion compensation).

The problem of the correct reconstruction of an image is also the topic of the paper by Y. Shkvarko et al. "Enhanced radar imaging in uncertain environment: A descriptive experiment design regularization approach." A new robust technique is developed by the authors for high-resolution reconstructive imaging, applied to enhanced remote sensing (RS) with an imaging array radar and/or a synthetic aperture radar (SAR), operating in an uncertain RS environment. The operational scenario uncertainties are associated with the unknown statistics of perturbations of the signal formation operator (SFO) in a turbulent medium, imperfect array calibration, finite dimensionality of measurements, uncontrolled antenna vibrations and, in the case of SAR, random platform trajectory deviations. In that paper, the authors propose new descriptive experiment design regularization (DEDR) approach to treat the uncertain radar image enhancement/reconstruction problems.

Another important research area for remote sensing is the application of satellite imagery for damage assessment. G. Trianni and P. Gamba, in "Damage detection from SAR imagery: application to the 2003 Algeria and 2007 Peru earthquakes," describe a method for fusing remotely sensed radar imagery with geographic databases. The method is illustrated by analysis of real radar imagery. It can provide rapid assessment of earthquake damage although further work is needed to improve the accuracy that can be achieved.

Real aperture radar also continues to play a fundamental role in remote sensing of the environment. P. L. Herselman et al. in "An analysis of X-band calibrated sea clutter and small boat reflectivity at medium to low grazing angles" show how accurate empirical modeling of sea clutter can provide the basis for inference of local sea conditions from remote low-grazing-angle radar returns. They have also analysed the coherent signatures of small boats, which interact in a complex way with the local clutter returns, and show how this information can be used to provide improved target detection methods.

The modeling of sea clutter is also the topic of the paper "Non-linear dynamics of sea clutter" by T. Field and S. Haykin. In their paper, the authors expand on the characterization of sea clutter as a nonlinear dynamic process, using the stochastic differential equation (SDE) theory. The stochastic dynamics of radar sea scattering are derived in terms of a pair of coupled stochastic differential equations for the received envelope and the radar crosssection (RCS). The analysis presented by the authors leads to the conclusion that, from both experimental and theoretical points of view, the dynamics of sea clutter are nonlinear with a consistent degree of nonlinearity that is determined by the sea state.

This special issue represents a small selection of current research. Nevertheless, this issue hascollected an interesting cross-section of papers representing work from 7 different countries. We would like to thank all the authors who contributed to this special issue and, in particular, the many anonymous referees who played such an important part.

Maria Greco

Simon Watts 

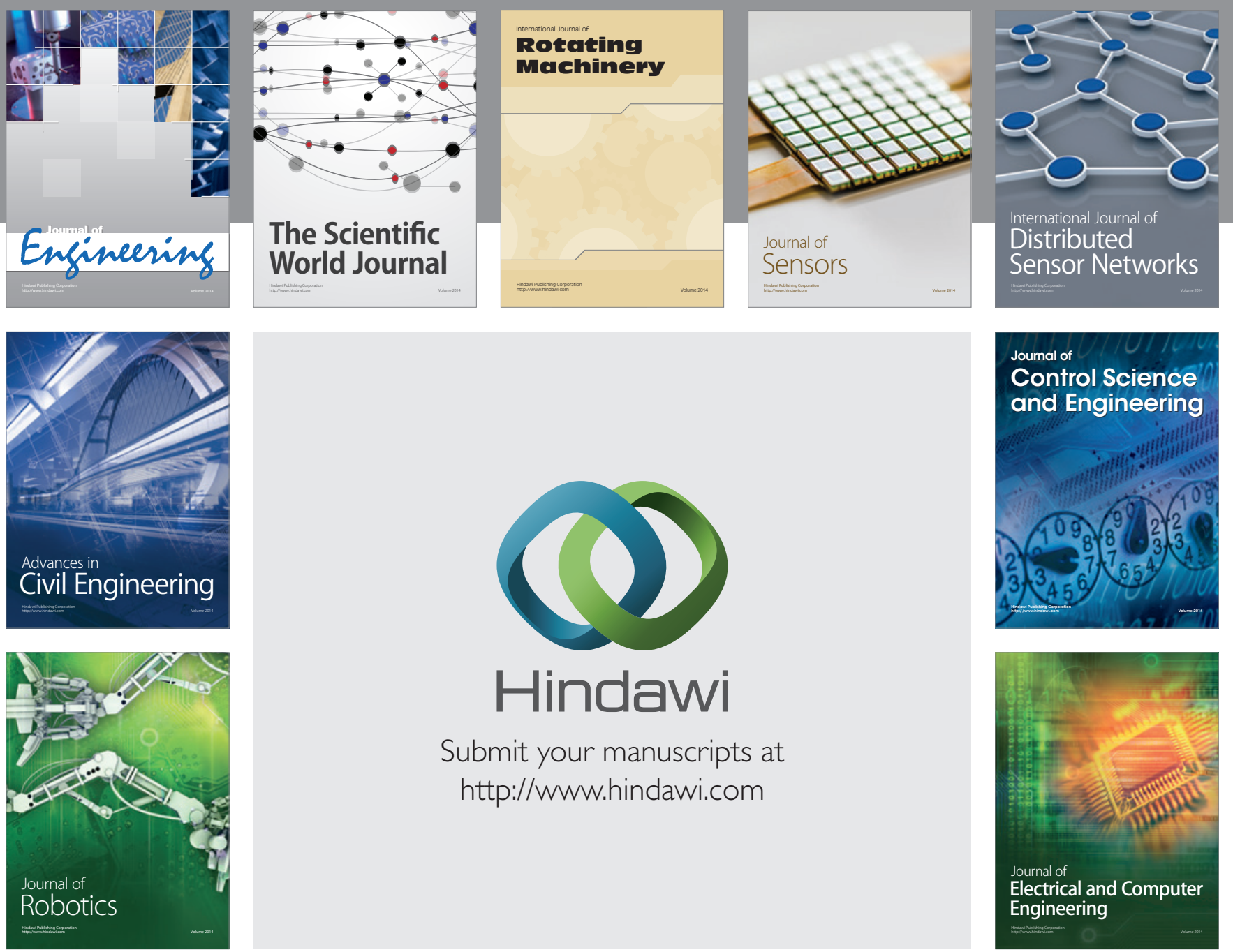

Submit your manuscripts at

http://www.hindawi.com
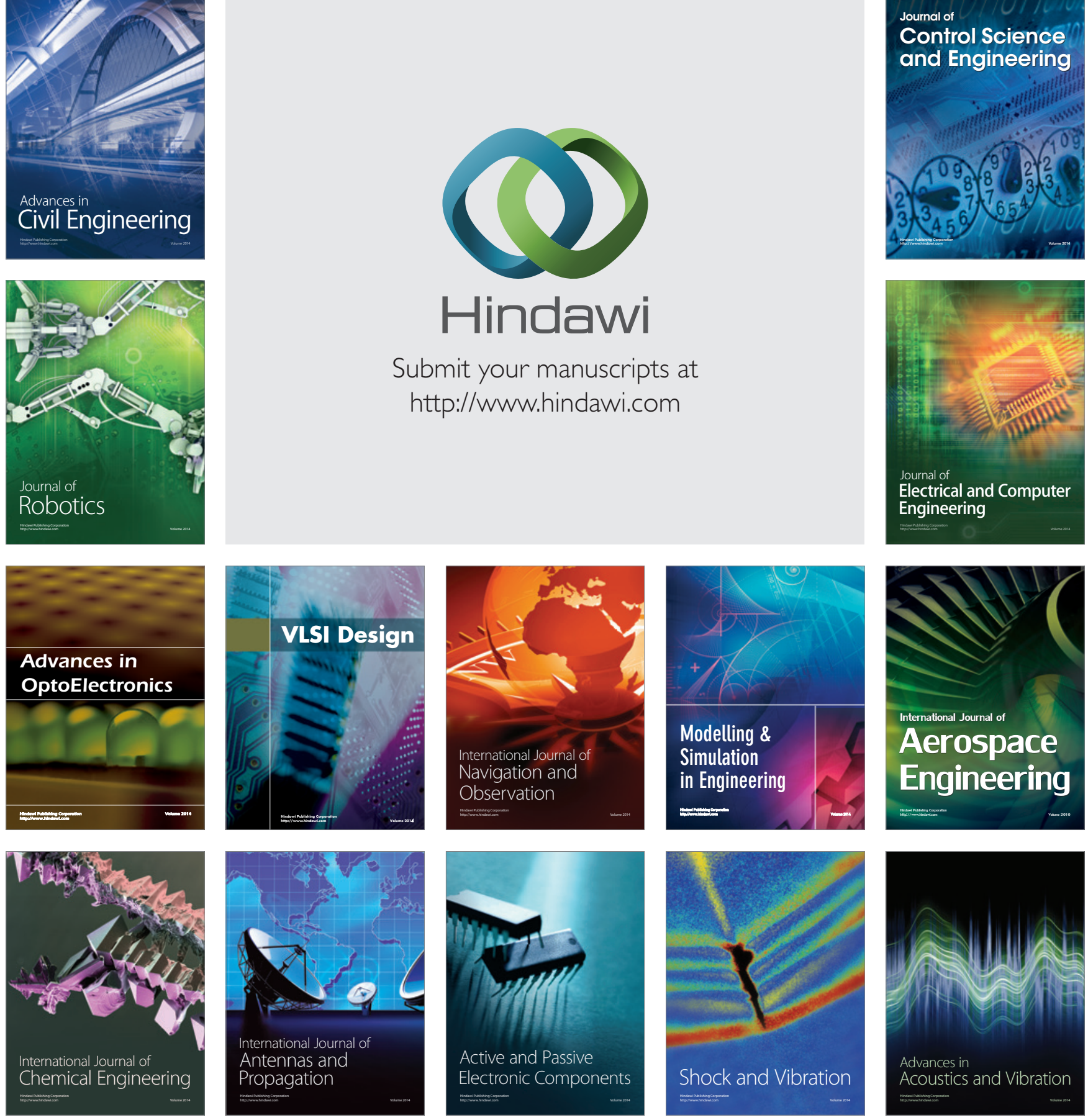